\title{
Influence of Amphiphilic Block Copolymers on Lyotropic Liquid Crystals in Water-Oil-Surfactant Systems
}

\author{
Christian Frank ${ }^{\dagger}$ Thomas Sottmann, $^{\ddagger}$ Cosima Stubenrauch, $*, \S$ \\ Jürgen Allgaier, ${ }^{\dagger}$ and Reinhard Strey
}

\begin{abstract}
Forschungszentrum Jülich GmbH, Institut für Festkörperforschung, D-52425 Jülich, Germany, Institut für Physikalische Chemie, Universität zu Köln, Luxemburger Strasse 116, D-50939 Köln, Germany, and Department of Chemical and Biochemical Engineering, University College Dublin, Belfield, Dublin 4, Ireland
\end{abstract}

Received June 3, 2005. In Final Form: July 20, 2005

\begin{abstract}
In ternary water-oil-nonionic alkyl polyglycol ether $\left(\mathrm{C}_{i} \mathrm{E}_{j}\right)$ microemulsions, an increase in efficiency is always accompanied by the formation of a lamellar $\left(\mathrm{L}_{\alpha}\right)$ phase. The addition of an amphiphilic block copolymer to the ternary base system increases the efficiency of the microemulsion drastically while suppressing-at least partly-the formation of the $\mathrm{L}_{\alpha}$ phase. However, amphiphilic block copolymers can be used not only to suppress the formation of lyotropic liquid crystals but also for the opposite effect, namely, to induce their formation. To understand to what extent the increase in efficiency is accompanied by the formation of lyotropic liquid crystals, we studied phase diagrams of water- $n$-alkane- $n$-alkyl polyglycol ethers $\left(\mathrm{C}_{i} \mathrm{E}_{j}\right)-\mathrm{PEPX}-\mathrm{PEOY}$ at a constant volume fraction of oil in the water/oil mixture. Using polymers of the poly(ethylene propylene)-copoly(ethylene oxide) type, with $M_{\mathrm{PEP}}=X \mathrm{~kg} \mathrm{~mol}^{-1}$ and $M_{\mathrm{PEO}}=Y \mathrm{~kg}$ $\mathrm{mol}^{-1}$, we determined phase diagrams as a function of the polymer concentration, size, and symmetry. Moreover, the influence of a particular polymer mixture was studied, which turned out to be the best system if both a high efficiency and a low tendency to form an $L_{\alpha}$ phase are needed.
\end{abstract}

\section{Introduction}

Lamellar $\left(\mathrm{L}_{\alpha}\right)$ phases and other lyotropic liquid crystals exist in many chemical and biological systems. They are well-known in binary water-surfactant and ternary water-oil- surfactant systems. In the former systems, the $\mathrm{L}_{\alpha}$ phase consists of surfactant bilayers surrounded by water, whereas in the latter systems, stacked monolayers separate oil and water domains. Liquid crystalline phases play a key role in many technical processes in which they are used as nanoreactors or templates for the synthesis of nanoparticles and mesoporous solid material. ${ }^{1-4}$ Moreover, $\mathrm{L}_{\alpha}$ phases form the membranes of biological cells. ${ }^{5}$ On the other hand, there are certain applications and processes in which the formation of lyotropic liquid crystals is undesirable because they are often highly viscous. Thus, the challenge is to control the stability of lyotropic liquid crystals in general and that of the $\mathrm{L}_{\alpha}$ phase in particular, that is, to induce or suppress them depending on the respective needs.

Looking at the phase diagrams of binary watersurfactant systems, one sees that the $\mathrm{L}_{\alpha}$ phase can expand over a broad concentration range, namely, from surfactant concentrations of more than $80 \mathrm{wt} \%$ down to surfactant concentrations of less than $1 \%$. Such expanded $\mathrm{L}_{\alpha}$ regions can be found using, for instance, the nonionic alkyl

* Corresponding author. E-mail: cosima.stubenrauch@ucd.ie; phone: +353-1-716-1923; fax: +353-1-716-1177.

Forschungszentrum Jülich GmbH.

$\doteqdot$ Universität zu Köln.

$\S$ University College Dublin.

(1) Attard, G. S.; Glyde, J. C.; Goltner, C. G. Nature 1995, 378, 366

(2) Göltner, C. G.; Berton, B.; Kramer, E.; Antonietti, M.Adv. Mater. 1999, 11, 395 .

(3) Kao, C.-P.; Lin, H. P.; Mou, C. Y. J. Phys. Chem. Solids 2001, 62, 1555.

(4) Han, B.-H.; Antonietti, M. J. Mater. Chem. 2003, 13, 1793.

(5) Structure and Dynamics of Membranes; Lipowsky, R., Sackmann, E., Eds.; Elsevier: New York, 1995. polyglycol ethers $\mathrm{C}_{12} \mathrm{E}_{5}$ ( $\mathrm{L}_{\alpha}$ down to $1 \%{ }^{6}$ ) or $\mathrm{C}_{10} \mathrm{E}_{4}$ ( $\mathrm{L}_{\alpha}$ down to $8 \%{ }^{7}$ ) as surfactants. Although the general phase behavior of binary water-surfactant systems is reviewed in detail by Laughlin, ${ }^{8}$ the control of the stability of the $\mathrm{L}_{\alpha}$ phase in these kinds of systems was discussed recently. ${ }^{7}$ The $\mathrm{L}_{\alpha}$ phase can also be observed over a broad concentration range in ternary water-oil-surfactant systems. ${ }^{9}$ When one considers the Gibbs phase triangle at the phase inversion temperature, it appears that the $\mathrm{L}_{\alpha}$ phase extends deep into the water and oil corners. ${ }^{10}$ These diluted $\mathrm{L}_{\alpha}$ phases consist of oil- and water-swollen surfactant bilayers, respectively. At equal volumes of water and oil, however, surfactant monolayers separate the two subdomains. Note that $\mathrm{L}_{\alpha}$ phases formed by monolayers are not as stable as those stabilized by bilayers, which is most likely due to the smaller bending rigidity of the monolayers. For example, at equal volume fractions of water and $n$-octane, at least $7 \mathrm{wt} \%$ of $\mathrm{C}_{12} \mathrm{E}_{5}$ molecules are needed to form a (monolayer) $\mathrm{L}_{\alpha}$ phase,${ }^{11}$ whereas a (bilayer) $\mathrm{L}_{\alpha}$ phase is already observed at $1 \mathrm{wt} \%$ in the corresponding water $-\mathrm{C}_{12} \mathrm{E}_{5}$ system. ${ }^{6}$

Upon dilution, both types of $\mathrm{L}_{\alpha}$ phases become unstable, which leads to the formation of isotropic phases. In the binary system, an $\mathrm{L}_{3}$ phase is formed in which a randomly oriented bilayer is found to divide the space into two equivalent water-continuous subvolumes. ${ }^{12}$ In the ternary system, however, a bicontinuous microemulsion is formed in which it is a randomly oriented monolayer that separates the continuous water- and oil-rich subvol-

(6) Strey, R. Ber. Bunsen-Ges. Phys. Chem. 1996, 100, 182

(7) Stubenrauch, C.; Burauer, S.; Strey, R.; Schmidt, C. Liq. Cryst. 2004, 31, 39.

(8) Laughlin, R. G.Aqueous Phase Behavior of Surfactants; Academic Press: New York, 1994.

(9) Kahlweit, M.; Strey, R.; Firman, P. J. Phys. Chem. 1986, 90, 671.

(10) Olsson, U.; Würz, U.; Strey, R. J. Phys. Chem. 1993, 97, 4535.

(11) Strey, R. Colloid Polym. Sci. 1994, 272, 1005.

(12) Porte, G.; Marignan, J.; Bassereau, P.; May, R. J. Phys. (Paris) 1988, 49,511 . 
umes. ${ }^{13,14}$ Golubovic, ${ }^{15}$ Morse ${ }^{16}$, and Gompper and Kroll 17 described this transition as a fluctuation-induced melting transition into a vesicle or a bicontinuous phase. They found out that the ratio of the bending elastic constants $\kappa$ and $\bar{\kappa}$ determines which of the two phases is formed. Furthermore, they predicted that a bicontinuous phase is stable only over a limited range of surfactant concentration. At high concentrations, it coexists with the $\mathrm{L}_{\alpha}$ phase, whereas a coexistence with an excess water phase (in the bilayer case) or excess water and oil phases (in the monolayer case) is predicted accurately at low concentration. It is, above all, the latter stability limit that plays an important role not only in the phase behavior of microemulsions but also in their technical applications ${ }^{18}$ because it determines the minimum surfactant concentration needed for a complete solubilization of equal volumes of water and oil and is thus a measure for the efficiency of the surfactant.

One of the central focuses of microemulsion formulation has always been the quest for high efficiency, that is, finding microemulsion systems in which the amount of surfactant necessary for solubilizing oil in water or vice versa is minimized. One way to increase the efficiency is to lengthen the hydrophobic alkyl chain of the surfactant. However, for low molecular weight surfactants, a gain in efficiency is accompanied by the formation of an $L_{\alpha}$ phase. As the extension of the $L_{\alpha}$ phase grows faster than the extension of the one-phase microemulsion, it finally dominates the phase diagram. ${ }^{9}$ Thus, the formulation of microemulsions with low molecular weight surfactants at reasonably low surfactant concentrations is a challenging task. Recently, we found that adding amphiphilic block copolymers to microemulsions significantly increases the efficiency in solubilizing water and oil. ${ }^{19-21}$ On the basis of the results obtained from phase diagrams and neutron scattering experiments, the increase in efficiency has been interpreted as an increase in the bending elastic constants, ${ }^{22-25}$ which, in turn, is caused by the adsorption of the amphiphilic block copolymers at the oil/water interface. According to the results published in ref 20, the presence of these particular amphiphilic block copolymers not only increases the efficiency of the microemulsion but also seems to suppress the formation of the $\mathrm{L}_{\alpha}$ phase. However, in a follow-up paper it was shown that this only holds true for very low polymer concentrations. ${ }^{26}$ At higher polymer concentrations a wide variety of lyotropic liquid

(13) Olsson, U.; Shinoda, K.; Lindman, B. J. Phys. Chem. 1986, 90, 4083.

(14) Jahn, W.; Strey, R. J. Phys. Chem. 1988, 92, 2294.

(15) Golubovic, L. Phys. Rev. E 1994, 50, 2419

(16) Morse, D. C. Phys. Rev. E 1994, 50, 2423

(17) Gompper, G.; Kroll, D. M. Phys. Rev. Lett. 1998, 81, 2284.

(18) Sottmann, T.; Strey, R. Microemulsions. In Fundamentals of Interface and Colloid Science; Lyklema, J., Ed.; Academic Press: New York, 2005; Vol. V.

(19) Jakobs, B.; Sottmann, T.; Strey, R.; Allgaier, J.; Willner, L.; Richter, D. Method for Increasing the Efficiency of Surfactants with Simultaneous Suppression of Lamellar Mesophases and Surfactants with an Additive Added Thereto. U.S. Patent 6,677,293 B1, 2004.

(20) Jakobs, B.; Sottmann, T.; Strey, R.; Allgaier, J.; Willner, L.; Richter, D. Langmuir 1999, 15, 6707.

(21) Sottmann, T. Curr. Opin. Colloid Interface Sci. 2002, 7, 57.

(22) Endo, H.; Allgaier, J.; Gompper, G.; Jakobs, B.; Monkenbusch,

M.; Richter, D.; Sottmann, T.; Strey, R. Phys. Rev. Lett. 2000, 85, 102.

(23) Endo, H.; Mihailescu, M.; Monkenbusch, M.; Allgaier, J.;

Gompper, G.; Richter, D.; Jakobs, B.; Sottmann, T.; Strey, R.; Grillo, I. J. Chem. Phys. 2001, 115, 580 .

(24) Gompper, G.; Endo, H.; Mihailescu, M.; Allgaier, J.; Monkenbusch, M.; Richter, D.; Jakobs, B.; Sottmann, T.; Strey, R. Europhys. Lett. 2001, 56, 683 .

(25) Gompper, G.; Richter, D.; Strey, R. J. Phys.: Condens. Matter 2001, 33, 9055 .

(26) Stubenrauch, C.; Frank, C.; Strey, R.; Burgemeister, D. Langmuir 2002, 18, 5027. crystals is formed, including the normal hexagonal $\left(\mathrm{H}_{1}\right)$, the $\mathrm{L}_{\alpha}$, and the reverse hexagonal $\left(\mathrm{H}_{2}\right)$ phases. Thus, as with microemulsions without amphiphilic block copolymers, the increasing efficiency is accompanied by an increasing tendency to form an $\mathrm{L}_{\alpha}$ phase. On the basis of these results and the above-mentioned theoretical considerations, ${ }^{15-17}$ the use of block copolymers to tune the efficiency of the microemulsion and the stability of the $\mathrm{L}_{\alpha}$ phase independently from each other does not seem to be very promising. However, what has not been taken into consideration yet is the fact that it is not only the polymer concentration but also the polymer structure (i.e., polymer size and symmetry) that can be varied. Moreover, mixtures of polymers offer a wide variety of parameters to examine.

This paper addresses the question of to what extent the increase in efficiency is accompanied by the formation of lyotropic liquid crystals. Although the role of the nonionic alkyl polyglycol ether surfactant $\left(\mathrm{C}_{i} \mathrm{E}_{j}\right)$ will be addressed, the focus is on the influence that different block copolymers have on the phase behavior of the ternary base system water- $n$-decane $-\mathrm{C}_{10} \mathrm{E}_{4}$. The aim is to adjust the properties of the amphiphilic surfactant/polymer mixture so that the efficiency-boosting effect can be optimized while the formation of lyotropic liquid crystals is simultaneously controlled. To monitor the relationship between increasing efficiency and the formation of lyotropic liquid crystals, we determined phase diagrams as a function of (a) the surfactant chain length, (b) the polymer concentration, (c) the polymer size, and (d) the polymer symmetry. Moreover, the influence of a particular polymer mixture will be presented and discussed.

\section{Experimental Section}

The investigated systems consisted of water, $n$-alkane, nonionic alkyl polyglycol ether surfactants $\left(\mathrm{C}_{i} \mathrm{E}_{j}\right)$, and amphiphilic block copolymers of the poly(ethylenepropylene)-copoly(ethyleneoxide) type. These polymers have structures that are similar to those of the $\mathrm{C}_{i} \mathrm{E}_{j}$ surfactants but have larger molar masses and are abbreviated PEPX-PEOY, in which X and Y denote the molecular weights of each block in kilograms/mole. A detailed description of their synthesis can be found in refs 27 and 28. The mixtures were prepared with deionized, twice-distilled water. The oils were purchased from Sigma Aldrich (Steinheim, Germany) and the surfactants were purchased from Bachem (Bubendorf, Switzerland) and Fluka (Buchs SG, Switzerland), both with a purity greater than $98 \%$. All components were used without further purification. The masses of the components water (A), oil (B), surfactant $(\mathrm{C})$, and polymer (D) are denoted as $m(\mathrm{~A}), m(\mathrm{~B}), m(\mathrm{C})$, and $m(\mathbf{D})$, respectively. The corresponding volumes of water and oil are denoted as $V(\mathrm{~A})$ and $V(\mathrm{~B})$. The composition of the samples is given by the volume fraction of oil in the water/oil mixture:

$$
\phi=\frac{V(\mathrm{~B})}{V(\mathrm{~B})+V(\mathrm{~A})}
$$

the overall mass fraction of the surfactant/polymer mixture:

$$
\gamma=\frac{m(\mathrm{C})+m(\mathrm{D})}{m(\mathrm{~A})+m(\mathrm{~B})+m(\mathrm{C})+m(\mathrm{D})}
$$

and the polymer mass fraction in the surfactant/polymer mixture:

$$
\delta=\frac{m(\mathrm{D})}{m(\mathrm{C})+m(\mathrm{D})}
$$

To determine the phase diagrams, the samples were weighted into test tubes and sealed. All of the samples were prepared at

(27) Allgaier, J.; Poppe, A.; Willner, L.; Richter, D. Macromolecules 1997, 30, 1582

(28) Poppe, A.; Willner, L.; Allgaier, J.; Stellbrink, J.; Richter, D. Macromolecules 1997, 30, 7462. 

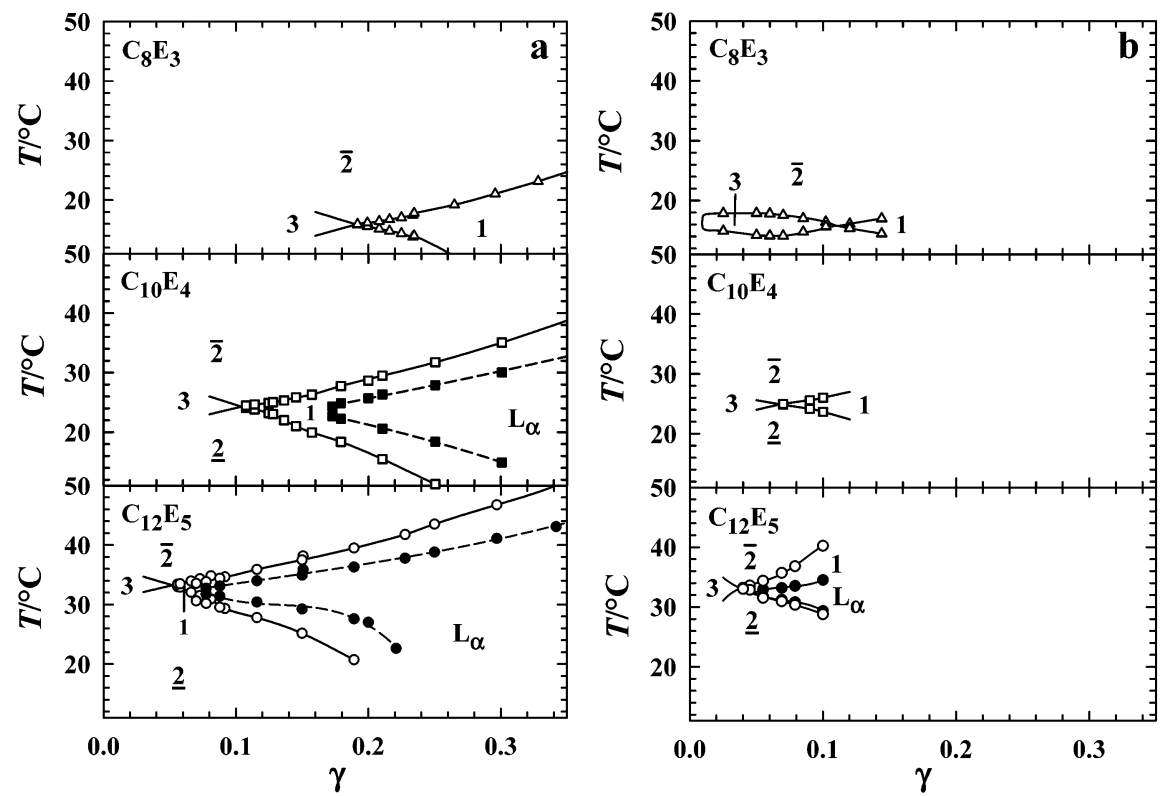

Figure 1. (a) Phase diagrams of different water- $n$-octane $-\mathrm{C}_{i} \mathrm{E}_{j}$ systems at a constant water-to-oil ratio of $\phi=0.5$. The increasing efficiency of the surfactant $\left(\mathrm{C}_{8} \mathrm{E}_{3}<\mathrm{C}_{10} \mathrm{E}_{4}<\mathrm{C}_{12} \mathrm{E}_{5}\right)$ is accompanied by an increasing expansion of the neighboring $\mathrm{L}_{\alpha}$ phase. (b) Phase diagrams of the same ternary systems shown in panel a after the amphiphilic block copolymer PEP5-PEO5 was added. A polymer mass fraction in the surfactant/polymer mixture of $\delta=0.05$ increases the efficiency drastically without significantly influencing the corresponding temperature $\tilde{T}$ of the $X$-point. Data for the system water $-n$-octane $-\mathrm{C}_{10} \mathrm{E}_{4}-\mathrm{PEP} 5-\mathrm{PEO} 5$ were taken from ref 30. Phase boundaries of the microemulsions (open symbols) and the liquid crystalline phases (filled symbols) are shown.

$\phi=0.5$. The determination of the phase behavior was carried out in a water bath with temperature control up to $\pm 0.02 \mathrm{~K}$. The samples were investigated at a given composition as a function of temperature. The occurring phases are characterized by visual inspection in transmitted light. Crossed polarizers were used to detect the presence of anisotropic phases. All phase boundaries were found to be reversible. The main problem occurred in connection with the regions denoted as 2 and $\overline{2}$ because the phase separation in these regions was very slow (at least a couple of weeks). These regions were not studied in detail; therefore we can exclude neither the coexistence of more than two phases nor the presence of liquid crystalline phases. The nature of the lyotropic liquid crystals was determined by polarization microscopy, ${ }^{2} \mathrm{H}$ NMR spectroscopy, ${ }^{26}$ and small angle neutron scattering (SANS). Moreover, the areas in the phase diagrams denoted as $\mathrm{I}_{1}$ and $\mathrm{I}_{2}$ are assumed to be cubic liquid crystals because the solutions are clear, isotropic, highly viscous, and are not stirrable. A detailed analysis and discussion of the microstructure will be presented in a follow-up paper.

\section{Results}

3.1. The Efficiency-Boosting Effect. The insight that we have gained thus far into the phase behavior of ternary water $-n$-alkane $-\mathrm{C}_{i} \mathrm{E}_{j}$ systems is reviewed in ref 18 . Here we determined that a useful way to characterize the phase behavior of these systems is to perform a section through the phase prism at a constant water-to-oil ratio of $\phi=0.5$, that is, at equal volume fractions of water and oil. Within such a temperature versus surfactant mass fraction section $[T(\gamma)]$, the phase boundaries resemble the shape of a "fish" (ref 18 and references therein). At low temperatures, an oil-in-water microemulsion coexists with an excess oil phase (denoted as 2), whereas, at high temperatures, a water-in-oil microemulsion coexists with an excess aqueous phase (denoted as $\overline{2}$ ). At intermediate temperatures, either a three-phase (denoted as 3 ) or a one-phase (denoted as 1) region is observed depending on the surfactant concentration. In the three-phase region, a bicontinuous microemulsion coexists with both an oil and an aqueous excess phase. As the surfactant concentration increases, the amount of the excess phases decreases until, finally, the one-phase region is reached. The minimum surfactant concentration at which equal volumes of water and oil are solubilized in one phase, that is, where the three-phase and the one-phase regions meet, is denoted as $\tilde{\gamma}$ at the temperature $\tilde{T}$. This particular point of the phase diagram is referred to as the $X$-point and is a useful measure for the efficiency of a surfactant. ${ }^{18}$ As the $\tilde{\gamma}$ value decreases, the efficiency of the surfactant increases.

It is well-known that the efficiency of a surfactant mainly depends on the surfactant's chain length. To increase the efficiency of a $\mathrm{C}_{i} \mathrm{E}_{j}$ surfactant for a given water-oil system, one has to increase the hydrophobic chain length while maintaining a constant headgroup size. Note that a decrease in the headgroup size while maintaining a constant chain length also increases the efficiency. However, the influence of the chain length is much more pronounced than that of the headgroup size, as is clearly demonstrated in Figure 1a in which $T(\gamma)$ sections of the $\mathrm{H}_{2} \mathrm{O}-n$-octane $-\mathrm{C}_{i} \mathrm{E}_{j}$ systems at $\phi=0.5$ are shown. Although the headgroup size increases from $\mathrm{E}_{3}$ to $\mathrm{E}_{5}$, the efficiency of the surfactant increases in the order $\mathrm{C}_{8} \mathrm{E}_{3}<$ $\mathrm{C}_{10} \mathrm{E}_{4}<\mathrm{C}_{12} \mathrm{E}_{5}$. Thus, an increase of the hydrophobic chain by two $\mathrm{CH}_{2}$ groups results in an enormous efficiency increase (see refs 18 and 29 for further details). Simultaneously, an $\mathrm{L}_{\alpha}$ phase [surrounded by a two-phase coexistence region (not shown)] appears, which is embedded in the homogeneous microemulsion phase. Upon increasing the surfactant chain length further, one sees that the $\mathrm{L}_{\alpha}$ phase extends almost across the entire onephase region. In the case of $\mathrm{C}_{12} \mathrm{E}_{5}$, for example, the concentration range of the bicontinuous microemulsion is reduced to less than $2 \mathrm{wt} \%$.

What occurs when parts of the surfactant are replaced by the amphiphilic block copolymer PEP5-PEO5 is seen in Figure $1 b$, in which the $T(\gamma)$ sections of the $\mathrm{H}_{2} \mathrm{O}-n$ octane $-\mathrm{C}_{i} \mathrm{E}_{j}-\mathrm{PEP} 5-\mathrm{PEO} 5$ systems at $\phi=0.5$ and a polymer mass fraction of $\delta=0.05$ in the surfactant/polymer mixture are shown. Comparing Figure 1, panels a and b,

(29) Burauer, S.; Sachert, T.; Sottmann, T.; Strey, R. Phys. Chem. Chem. Phys. 1999, 1, 4299. 


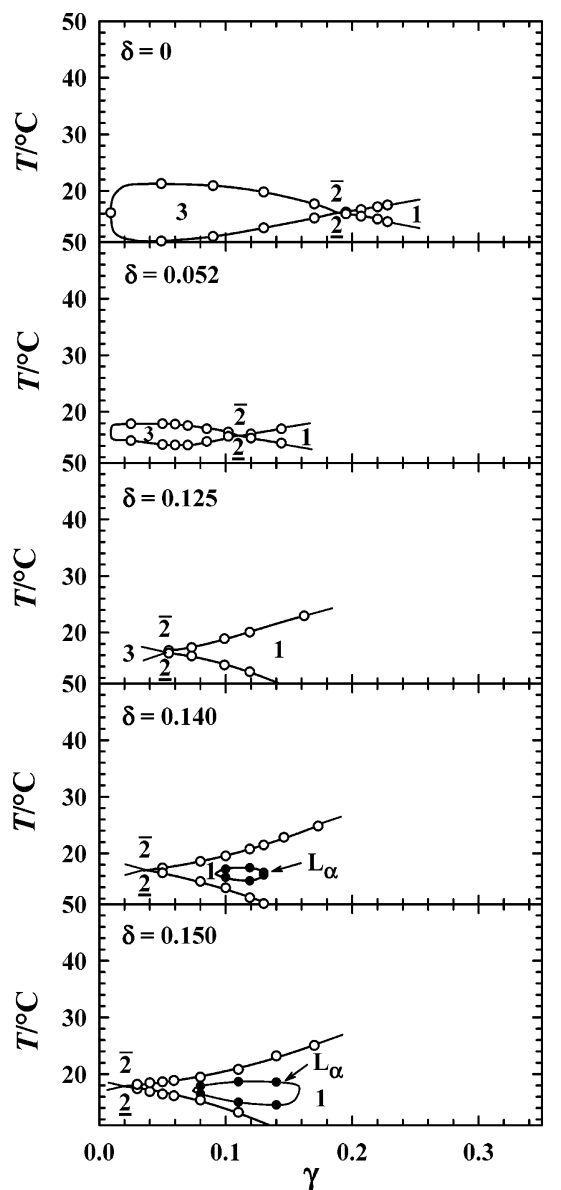

Figure 2. Phase diagrams of the system $\mathrm{H}_{2} \mathrm{O}-n$-octane $-\mathrm{C}_{8} \mathrm{E}_{3}-$ PEP5-PEO5 at a constant water-to-oil ratio of $\phi=0.5$ as a function of the polymer mass fraction $\delta$. With increasing $\delta$, the efficiency increases, and the expansion of the three-phase region (fish body) decreases. At a polymer mass fraction in the surfactant/polymer mixture of $\delta \geq 0.140$, an $\mathrm{L}_{\alpha}$ island appears. Phase boundaries of the microemulsions (open symbols) and the liquid crystalline phases (filled symbols) are shown.

it can be seen that the addition of PEP5-PEO5 leads to a reduction of $\tilde{\gamma}$ in all three systems. In other words, the efficiency of the surfactant/polymer mixture is significantly enhanced compared to that of the polymer-free system. This effect is known as "efficiency boosting", which was first described only six years ago. ${ }^{19,20}$ Furthermore, it is worth mentioning that adding the block copolymer reduces $\tilde{\gamma}$ without significantly influencing the corresponding temperature $\tilde{T}$ of the $X$-point.

3.2. Role of the Polymer Concentration. The role of the polymer concentration with respect to efficiency boosting as well as to the formation of lyotropic liquid crystals in general and the $\mathrm{L}_{\alpha}$ phase in particular is demonstrated in Figures 2 and 3. In Figure 2, phase diagrams of the system water- $n$-octane $-\mathrm{C}_{8} \mathrm{E}_{3}-\mathrm{PEP} 5-$ PEO5 at a constant oil-to-water volume fraction of $\phi=0.5$ are shown as a function of the polymer mass fraction $\delta$. Note that the phase diagram of the ternary base system $(\delta=0)$ is the same as that seen in Figure 1a. However, in contrast to Figure 1a, the three-phase region is shown.

Starting from the ternary base system, the replacement of $\mathrm{C}_{8} \mathrm{E}_{3}$ by PEP5-PEO5 first leads to a continuous shift of the $X$-point toward lower $\tilde{\gamma}$ values while the corresponding temperature $\tilde{T}$ remains almost unaffected. At the highest polymer mass fraction investigated in this study (i.e., at $\delta=0.150$ ) only 2 wt $\%$ of the $\mathrm{C}_{8} \mathrm{E}_{3} / \mathrm{PEP} 5-$ PEO5 mixture is needed to solubilize equal amounts of water and oil. Another important influence the polymer

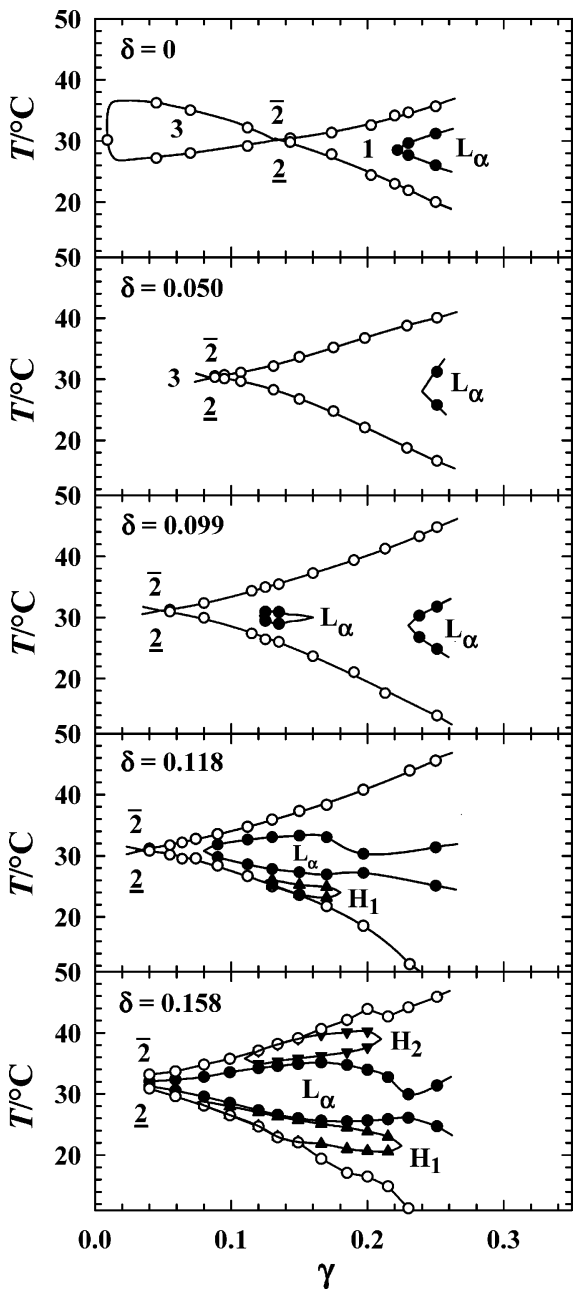

Figure 3. Phase diagrams of the system $\mathrm{H}_{2} \mathrm{O}-n$-decane$\mathrm{C}_{10} \mathrm{E}_{4}-\mathrm{PEP} 5-\mathrm{PEO} 5$ at a constant water-to-oil ratio of $\phi=0.5$ as a function of the polymer mass fraction $\delta$ in the surfactant/ polymer mixture. With increasing $\delta$, the efficiency increases, and the extension of the three-phase region (fish body) decreases. Whereas the $\mathrm{L}_{\alpha}$ phase formed at high $\gamma$ remains nearly unaffected, additional lyotropic mesophases appear as $\delta$ increases, namely, a second $\mathrm{L}_{\alpha}$ phase at $\delta \geq 0.099$ and hexagonal phases at $\delta \geq 0.118$. At $\delta=0.158$, the inverse hexagonal phase $\mathrm{H}_{2}$ appears for the first time, which was identified by ${ }^{2} \mathrm{H} \mathrm{NMR} .{ }^{26}$ The fish-tail point at $\delta=0.158$ could not be measured because the sample was turbid because of the large domain size. Data for $\delta=0,0.050$, and 0.118 were taken from ref 32. Phase boundaries of the microemulsions (open symbols) and the liquid crystalline phases (filled symbols) are shown.

has on the phase diagram is seen at $\delta \geq 0.14$. At these polymer mass fractions an $\mathrm{L}_{\alpha}$ island appears at low surfactant concentrations. Note that the polymer-free system forms an $\mathrm{L}_{\alpha}$ phase only at surfactant concentrations higher than 0.35 (not shown), whereas the polymerinduced $\mathrm{L}_{\alpha}$ phase is already formed at $\gamma<0.10$. Similar observations are made for the system water $-n$-decane$\mathrm{C}_{10} \mathrm{E}_{4}-\mathrm{PEP} 5-\mathrm{PEO}$. In Figure 3, the respective phase diagrams at $\phi=0.5$ are shown as a function of the polymer mass fraction $\delta$.

Analogous to the results obtained for $\mathrm{C}_{8} \mathrm{E}_{3}$, the replacement of $\mathrm{C}_{10} \mathrm{E}_{4}$ by PEP5-PEO5 significantly increases the efficiency without affecting the corresponding temperature $\tilde{T}$. At $\delta=0.118$ only $4 \mathrm{wt} \%$ of the $\mathrm{C}_{10} \mathrm{E}_{4} / \mathrm{PEP} 5-\mathrm{PEO} 5$ mixture is needed to solubilize equal amounts of water and oil. As is seen in Figure 3, the efficiency can be increased even further. However, the determination of the phase boundaries becomes increasingly difficult-and finally impossible-because of the extremely strong light 


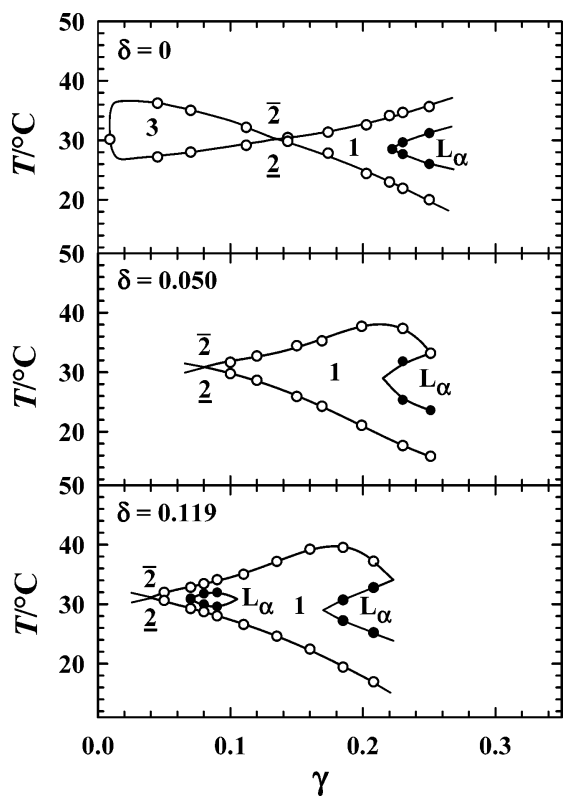

Figure 4. Phase diagrams of the system $\mathrm{H}_{2} \mathrm{O}-n$-decane$\mathrm{C}_{10} \mathrm{E}_{4}-\mathrm{PEP} 10-\mathrm{PEO} 10$ at a constant water-to-oil ratio of $\phi=$ 0.5 as a function of the polymer mass fraction $\delta$ in the surfactant/ polymer mixture. With increasing $\delta$, the efficiency increases, and the expansion of the three-phase region (fish body) decreases. The position of the $\mathrm{L}_{\alpha}$ phase is slightly shifted toward lower $\gamma$ until a second $\mathrm{L}_{\alpha}$ phase appears at $\delta=0.119$ as a small island. Phase boundaries of the microemulsions (open symbols) and the liquid crystalline phases (filled symbols) are shown.

scattering of the microemulsion. Moreover, the large stability of the $\mathrm{L}_{\alpha}$ phase allows the one-phase microemulsion to exist only in small concentration and temperature ranges. The extended $L_{\alpha}$ region seen at $\delta=0.158$ evolves from a small $\mathrm{L}_{\alpha}$ island that appears at concentrations around $\delta=0.099$. Whereas the $\mathrm{L}_{\alpha}$ phase at high surfactant concentrations is nearly unaffected by the polymer concentration $\delta$, the $\mathrm{L}_{\alpha}$ island at low $\gamma$ increases with increasing $\delta$ in both directions, that is, toward lower and higher surfactant concentrations. At $\delta=0.118$, the $\mathrm{L}_{\alpha}$ island and the $L_{\alpha}$ phase at high surfactant concentrations meet each other so that an extended $\mathrm{L}_{\alpha}$ region is formed. The difference between the two $\mathrm{L}_{\alpha}$ regions, which finally fuse with each other, will be addressed in connection with Figure 9. Increasing $\delta$ even further, in addition to the diluted $\mathrm{L}_{\alpha}$ phase, hexagonal phases appear both above and below the $L_{\alpha}$ region so that the phase sequence $\mathrm{H}_{1} \rightarrow$ $\mathrm{L}_{\alpha} \rightarrow \mathrm{H}_{2}$ can be observed with increasing temperature. ${ }^{26}$

3.3. Role of Polymer Size and Polymer Symmetry. Polymer Size. In the following section, the influence of polymer size and polymer symmetry will be demonstrated. For the sake of clarity, from now on we will always refer to the same ternary base system, namely, water- $n$ decane $-\mathrm{C}_{10} \mathrm{E}_{4}$. To investigate the influence of the polymer size on the phase behavior, we replaced PEP5-PEO5 with PEP10-PEO10, which means that the molecular weight of both the hydrophilic and the hydrophobic part was doubled. In Figure 4 , the $T(\gamma)$ sections of the $\mathrm{H}_{2} \mathrm{O}-n$ decane $-\mathrm{C}_{10} \mathrm{E}_{4}-\mathrm{PEP} 10-\mathrm{PEO} 10$ system at $\phi=0.5$ and $\delta$ $=0,0.050$, and 0.119 are shown.

Comparing Figure 4 with Figure 3, one sees that adding either PEP5-PEO5 or PEP10-PEO10 does not make a significant difference as far as a mass fraction of $\delta=0.05$ is concerned. The increase in efficiency is approximately the same, the temperature $\tilde{T}$ is not changed, and the position of the $\mathrm{L}_{\alpha}$ phase is hardly affected in both systems. Note that equal polymer mass fractions are compared, which means that the number of PEP10-PEO10 molecules is half the number of PEP5-PEO5 molecules. We will come back to this point in Section 4.3. Analogous to the observations made at $\delta=0.05$, at $\delta=0.119$, no differences with regard to the efficiency increase are seen between the PEP5-PEO5 and the PEP10-PEO10 systems. However, at $\delta=0.119$, the size of the polymer affects the extension of the lyotropic liquid crystals. Although one extended $L_{\alpha}$ region and a hexagonal phase were observed in the respective phase diagram of the PEP5PEO5 system, the phase diagram of the PEP10-PEO10 system formed two unconnected $L_{\alpha}$ regions and no hexagonal phase. Thus, the polymer size does not influence the efficiency-boosting effect; however, an influence on the formation of the liquid crystalline phases is obvious: an increase in the polymer size results in a suppression of the liquid crystalline phases $\mathrm{L}_{\alpha}$ and $\mathrm{H}_{1}$. This is a very important result because we now have a tuning parameter, namely, the polymer size, with which the efficiency of the microemulsion and the formation of liquid crystalline phases can be affected separately.

Polymer Symmetry. To study the influence of polymer symmetry, polymers of the same molecular weight but different hydrophilic and hydrophobic block size were added to the base system $\mathrm{H}_{2} \mathrm{O}-n$-decane- $\mathrm{C}_{10} \mathrm{E}_{4}$. Therefore, the symmetric PEP10-PEO10 system that was studied before was replaced by the more hydrophilic polymer PEP5-PEO15 and the more hydrophobic polymer PEP15-PEO5. The phase diagrams of the PEP5-PEO15 and PEP15-PEO5 systems at different polymer mass fractions $\delta$ in the surfactant/polymer mixture are shown in Figure 5a and Figure 5b, respectively.

A comparison of the phase diagrams at equal $\delta$ reveals that the efficiency-boosting effect of the polymer does not depend on the symmetry. However, the temperature $\tilde{T}$ of the $X$-point is shifted slightly toward higher temperatures in the hydrophilic PEP5-PEO15 system and slightly toward lower $\tilde{T}$ values for the hydrophobic PEP15-PEO5. Considering the tendency to form lyotropic liquid crystals, one sees that, in both cases, an $\mathrm{L}_{\alpha}$ island is formed at $\delta$ $\geq 0.119$ and additional phases appear at slightly higher $\delta$. The difference is the nature of the lyotropic liquid crystals. Whereas with PEP5-PEO15, the inverse phases $\mathrm{H}_{2}$ and $\mathrm{I}_{2}$ are formed, the $\mathrm{H}_{1}$ and the $\mathrm{I}_{1}$ phases appear in the PEP15-PEP5 system. Surprisingly, in both systems, the corresponding inverted lyotropic liquid crystalline phase are missing $\left(\mathrm{H}_{1}\right.$ and $\mathrm{I}_{1}$ in the PEP5-PEO15 system and $\mathrm{H}_{2}$ and $\mathrm{I}_{2}$ in the PEP15-PEP5 system). However, their absence can be explained by assuming that the phase behavior of liquid crystals is less temperature dependent than that of microemulsions. If this is true, the missing liquid crystalline phases should be present in the twophase regions 2 and $\overline{2}$, respectively, which we did not study in detail.

In conclusion, one can say that an inversion of the symmetry, that is, a change from PEP5-PEO15 to PEP15-PEO5, influences neither the increase in efficiency nor the extent to which lyotropic liquid crystals are formed. What mainly differs is the nature of the lyotropic liquid crystals existing within the one-phase region of the microemulsion. Once it was discovered that the size of the polymer is a tuning parameter that affects the efficiency of the microemulsion and the formation of liquid crystalline phase separately, the next step was to study how the size of one of the two polymer blocks influences the phase behavior. Therefore the molecular weight of one of the blocks was systematically increased by keeping the molecular mass of the other block and the polymer mass fraction $\delta$ in the surfactant/polymer mixture constant. As 

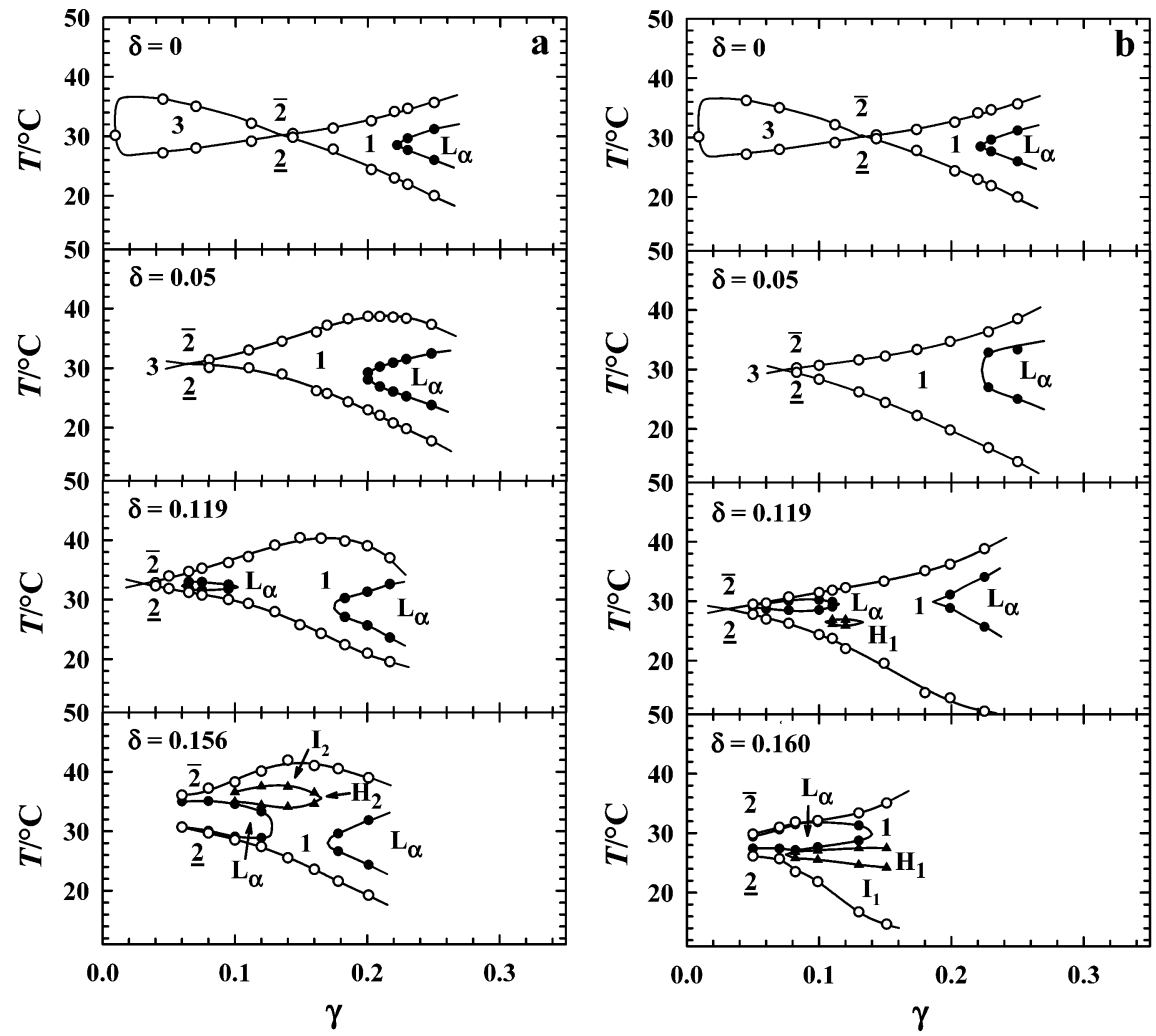

Figure 5. (a) Phase diagrams of the system $\mathrm{H}_{2} \mathrm{O}-n$-decane $-\mathrm{C}_{10} \mathrm{E}_{4}-\mathrm{PEP} 5-\mathrm{PEO} 15$ at a constant water-to-oil ratio of $\phi=0.5$ as a function of $\delta$. The increase of $\delta$ results in three noteworthy features. First, the temperature $\tilde{T}$ of the $X$-point increases slightly but significantly. Second, the $\mathrm{L}_{\alpha}$ phase at high $\gamma$ is nearly unaffected. Third, additional lyotropic mesophases, such as a second $\mathrm{L}_{\alpha}$ and an $\mathrm{H}_{2}$, appear (an $\mathrm{H}_{1}$ has not been detected but may be present in the 2 region). In addition, a highly viscous area was found (denoted as $\mathrm{I}_{2}$ because it may be cubic in comparison). (b) Phase diagrams of the system $\mathrm{H}_{2} \mathrm{O}-n$-decane- $\mathrm{C}_{10} \mathrm{E}_{4}-\mathrm{PEP} 15-\mathrm{PEO} 5$ at $\phi=0.5$ as a function of $\delta$. The phase diagrams are shifted slightly but significantly to lower temperatures. They are remarkably similar to those of the asymmetric counterpart PEP5-PEO15. Because of the interchanged block sizes at $\delta \geq 0.119$, an $\mathrm{H}_{1}$ appears (and an $\mathrm{H}_{2}$ may be present in the $\overline{2}$ region), and at $\delta=0.160$, a highly viscous area was found (denoted as $\mathrm{I}_{1}$ because it may be cubic). Phase boundaries of the microemulsions (open symbols) and the liquid crystalline phases (filled symbols) are shown.

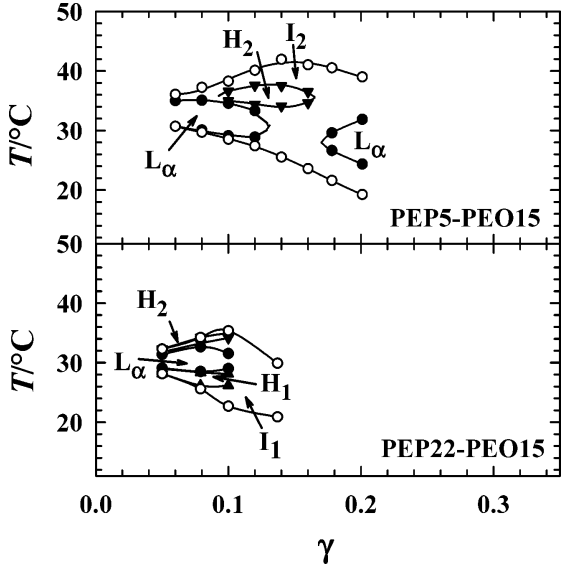

Figure 6. Phase diagrams of the systems $\mathrm{H}_{2} \mathrm{O}-n$-decane$\mathrm{C}_{10} \mathrm{E}_{4}-\mathrm{PEP} 5-\mathrm{PEO} 15$ and $\mathrm{H}_{2} \mathrm{O}-n$-decane- $\mathrm{C}_{10} \mathrm{E}_{4}-\mathrm{PEP} 22-$ PEO15 at a constant water-to-oil ratio of $\phi=0.5$ and a polymer mass fraction in the surfactant/polymer mixture of $\delta=0.16$. Whereas the PEP5-PEO15 system forms only the inverted lyotropic mesophases, the more hydrophobic PEP22-PEO15 system exhibits a nearly complete sequence of lyotropic mesophases, that is, $\mathrm{I}_{1}, \mathrm{H}_{1}, \mathrm{~L}_{\alpha}$, and $\mathrm{H}_{2}$. Phase boundaries of the microemulsions (open symbols) and the liquid crystalline phases (filled symbols) are shown.

mentioned above, equal mass fractions do not mean equal numbers of molecules because the molecular weights differ.

Size of the Hydrophobic Block. In Figure 6, the $T(\gamma)$ section of the $\mathrm{H}_{2} \mathrm{O}-n$-decane- $\mathrm{C}_{10} \mathrm{E}_{4}-\mathrm{PEP} 5-\mathrm{PEO} 15$ and
$\mathrm{H}_{2} \mathrm{O}-n$-decane- $\mathrm{C}_{10} \mathrm{E}_{4}-\mathrm{PEP} 22-\mathrm{PEO} 15$ systems at $\phi=$ 0.5 and a polymer mass fraction in the surfactant/polymer mixture of $\delta=0.16$ are shown (the phase diagram of the PEP5-PEO15 system is the same as that presented in Figure 5a).

Although the phase behavior could not be measured at low values of $\gamma$ because of the very strong light scattering, one can see that both systems are very efficient, that is, $\tilde{\gamma}$ values well below 0.04 are expected. With regard to the temperature, the phase behavior changes as expected: increasing the molar mass of the hydrophobic polymer block shifts the phase diagram to lower temperatures. Considering the tendency to form lyotropic liquid crystals, one sees that the PEP5-PEO15 system shows only the inverted lyotropic mesophases, whereas the more hydrophobic PEP22-PEO15 system exhibits a nearly complete sequence of lyotropic mesophases: $\mathrm{I}_{1}, \mathrm{H}_{1}, \mathrm{~L}_{\alpha}$, and $\mathrm{H}_{2}$. Only the inverted cubic phase $\mathrm{I}_{2}$ is missing.

Size of the Hydrophilic Block. In Figure 7, the phase diagrams of the system $\mathrm{H}_{2} \mathrm{O}-n$-decane $-\mathrm{C}_{10} \mathrm{E}_{4}-\mathrm{PEP} 22-$ PEOY at $\phi=0.5$ and a polymer mass fraction in the surfactant/polymer mixture of $\delta=0.16$ are shown for $\mathrm{Y}$ $=15,11$, and 4 .

Analogous to the phase diagrams shown in Figure 6, the phase behavior of these three systems could not be measured at low values of $\gamma$ because of the very strong light scattering. It is clearly seen, however, that the systems are very efficient and that lyotropic liquid crystals dominate the phase behavior at low mass fractions of surfactant and polymer. As was the case in Figure 6, as the hydrophobicity of the polymer increases, the phase 


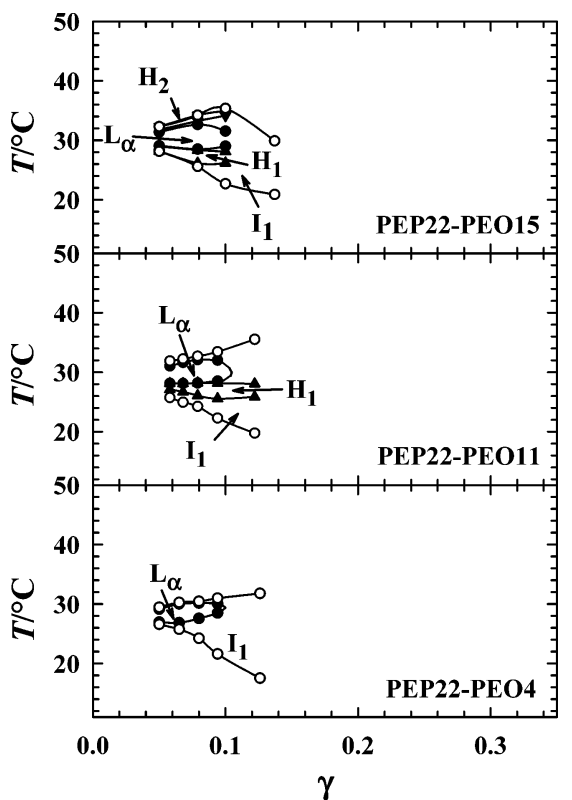

Figure 7. Phase diagrams of the systems $\mathrm{H}_{2} \mathrm{O}-n$-decane$\mathrm{C}_{10} \mathrm{E}_{4}-\mathrm{PEP} 22-\mathrm{PEOY}$ with $\mathrm{Y}=4,11$, and 15 at a constant water-to-oil ratio of $\phi=0.5$ and a polymer mass fraction of $\delta$ $=0.16$. As the size of the hydrophilic block PEOY decreases, the phase behavior shifts to lower temperatures. Furthermore, both of the hexagonal phases are suppressed, whereas the expansion of the cubic $I_{1}$ phase increases. Phase boundaries of the microemulsions (open symbols) and the liquid crystalline phases (filled symbols) are shown.

diagram shifts to lower temperatures. Therefore, the formation of the normal lyotropic liquid crystals $\mathrm{H}_{1}$ and $\mathrm{I}_{1}$ is increasingly favored. Again, this trend can be explained by assuming that the phase behavior of liquid crystals is less temperature dependent than that of microemulsions; therefore, the inverse liquid crystalline phases could be present in the two-phase region $\overline{2}$, which we did not study in detail (see also Experimental Section). Thus, the variety of observed phases decreases with increasing hydrophobicity, that is, decreasing hydrophilic block size. Whereas the sequence $\mathrm{I}_{1} \rightarrow \mathrm{H}_{1} \rightarrow \mathrm{L}_{\alpha} \rightarrow \mathrm{H}_{2}$ was found in the PEP22-PEO15 system, only the $\mathrm{L}_{\alpha}$ and a $\mathrm{I}_{1}$ phases were observed in the more hydrophobic PEP22$\mathrm{PEO} 4$ system. To conclude, one can say that the variation of the block sizes influences, first and foremost, the nature of the lyotropic liquid crystals that are observed. As the hydrophobicity of the polymer increases, the temperatures of the phase boundaries of the one-phase region decreases, and the normal liquid crystals such as $\mathrm{H}_{1}$ and $\mathrm{I}_{1}$ are more favored. We will come back to this point in Section 4.3.

3.4. Role of Polymer Mixtures. Last but not least, the use of polymer mixtures to independently tune the efficiency and the formation of lyotropic liquid crystals was studied. Figure 8 shows the phase diagrams of $\mathrm{H}_{2} \mathrm{O}-$ $n$-decane- $\mathrm{C}_{10} \mathrm{E}_{4}-\mathrm{PEP} 10-\mathrm{PEO} 10$ and $\mathrm{H}_{2} \mathrm{O}-n$-decane$\mathrm{C}_{10} \mathrm{E}_{4}-\mathrm{PEP} 5-\mathrm{PEO} 15 / \mathrm{PEP} 15-\mathrm{PEO} 5$ at $\delta=0.119$ and $\phi$ $=0.5$ for comparison. The polymer mixture consists of equal amounts of PEP5-PEO15 and PEP15-PEO5.

As is seen in Figure 8, the two phase diagrams are nearly equal with one exception: the mixed polymer system lacks the $\mathrm{L}_{\alpha}$ island at low surfactant concentrations. The determination of equal efficiencies and phase boundaries can be deduced from the results obtained thus far. First, it was shown in Section 3.3 that the polymer symmetry at a given polymer mass fraction and polymer molecular weight does not influence the efficiency (Figures 4 and 5). Second, the shapes of the phase boundaries seen in Figure 5 indeed suggest that mixing these two polymers evens

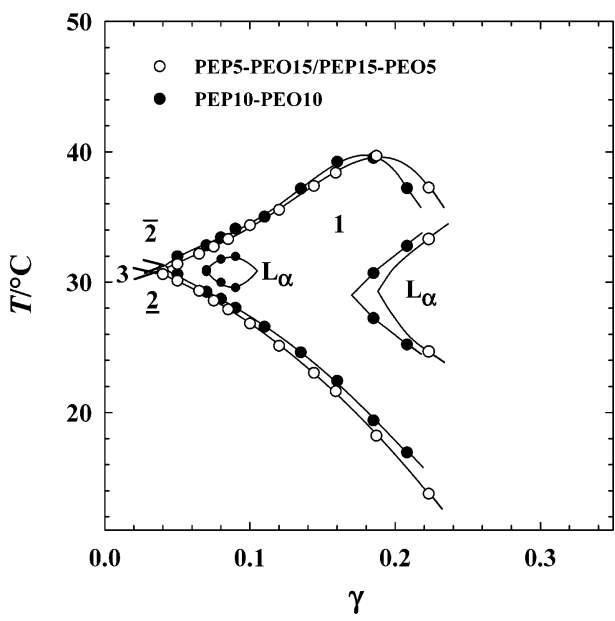

Figure 8. Phase diagrams of $\mathrm{H}_{2} \mathrm{O}-n$-decane $-\mathrm{C}_{10} \mathrm{E}_{4}-\mathrm{PEP} 10-$ PEO10 and $\mathrm{H}_{2} \mathrm{O}-n$-decane $-\mathrm{C}_{10} \mathrm{E}_{4}-(\mathrm{PEP} 5-\mathrm{PEO} 15 / \mathrm{PEP} 15-$ PEO5) at $\delta=0.119$ and $\phi=0.5$. The polymer mixture consists of equal amounts PEP5-PEO15 and PEP15-PEO5. The phase boundaries of the one-phase region and the $\mathrm{L}_{\alpha}$ phase at high $\gamma$ are nearly identical. Interestingly, the $\mathrm{L}_{\alpha}$ island at low $\gamma$ is not formed in the system containing the polymer mixture [for reasons discussed in the text and illustrated in Figure 10 (bottom)]. Phase boundaries of the microemulsions (open symbols) and the liquid crystalline phases (filled symbols) are shown.

out the opposite curvatures and results in phase boundaries similar to those of the symmetric PEP10-PEO10 system. What is unexpected, however, is the complete suppression of the $\mathrm{L}_{\alpha}$ island because, at $\delta=0.119$, an $\mathrm{L}_{\alpha}$ island of similar size is formed in the corresponding pure polymer systems, that is, in the systems containing PEP5PEO15 and PEP15-PEO5 (see Figure 5). An explanation of why mixing two polymers of inverse symmetry suppresses the formation of the $L_{\alpha}$ island will be given in Section 4.4. In conclusion, the mixing of asymmetric polymers of large molecular mass seems to be the most promising way to tune the phase behavior of microemulsion systems. By mixing appropriate polymers, one can not only achieve an enormous increase in efficiency, but can also control $\mathrm{L}_{\alpha}$ formation.

\section{Discussion}

4.1. Efficiency Boosting. To understand the mechanism behind the efficiency boosting of amphiphilic block copolymers, one has to keep in mind that the behavior of complex fluids is controlled by the properties of the amphiphilic film. According to the measured phase diagrams ${ }^{20,30}$ and to neutron scattering experiments, ${ }^{23,24}$ the adsorption of the amphiphilic block copolymer at the water/oil interface influences the interfacial properties significantly. Recently, the effect of polymers anchored to membranes has been calculated for Gaussian (ideal) chains. ${ }^{31}$ In the mushroom regime, the effective bending moduli $^{22}$ are found to be

$$
\kappa_{\mathrm{eff}}=\kappa+\frac{k_{\mathrm{B}} T}{12}\left(1+\frac{\pi}{2}\right) \sigma\left(R_{\mathrm{a}}^{2}+R_{\mathrm{b}}^{2}\right)
$$

(30) Strey, R.; Brandt, M.; Jakobs, B.; Sottmann, T. Stud. Surf. Sci. Catal. 2001, 132, 39.

(31) Hiergeist, C.; Lipowsky, R. J. Phys. II 1996, 6, 1465.

(32) Jakobs, B. Amphiphile Blockcopolymere als "Efficiency Booster" für Tenside: Entdeckung und Aufklärung des Effekts. InauguralDissertation, Universität zu Köln, 2001. 
and

$$
\bar{\kappa}_{\text {eff }}=\bar{\kappa}-\frac{k_{\mathrm{B}} T}{6} \sigma\left(R_{\mathrm{a}}{ }^{2}+R_{\mathrm{b}}{ }^{2}\right)
$$

in which $\kappa$ and $\bar{\kappa}$ are the bending moduli of the pure surfactant membrane, $R_{\mathrm{a}}$ and $R_{\mathrm{b}}$ are the end-to-end distances of the hydrophilic and hydrophobic block, respectively, and $\sigma$ is the number density of the block copolymer within the membrane. The stability limit of the $\mathrm{L}_{\alpha}$ and bicontinuous phases is defined as

$$
\ln \phi_{\mathrm{C}} \geq \frac{6 \pi}{5} \frac{\bar{\kappa}}{k_{\mathrm{B}} T}
$$

with $\phi_{\mathrm{C}}$ being the surfactant volume fraction. ${ }^{15-17}$ Combining eqs 5 and 6 leads to

$$
\ln \phi_{\mathrm{C}} \geq \ln \phi_{\mathrm{C}}^{0}-\Xi \sigma\left(R_{\mathrm{a}}^{2}+R_{\mathrm{b}}^{2}\right)
$$

in which $\phi_{\mathrm{C}}^{0}$ is the surfactant volume fraction at the fishtail point ( $X$-point) of the polymer-free system, and $\Xi=$ $\pi / 5=0.628$. Note that the factor found experimentally ${ }^{22}$ was $\Xi=1.54$, which is twice as large as that predicted, and this difference is discussed in ref 22 in terms of selfavoiding chains. Finally, the influence of the amphiphilic block copolymer on the "effective" spontaneous curvature is predicted ${ }^{25}$ to be

$$
c_{0, \mathrm{eff}}(T)=c_{0}(T)+\frac{1}{4} \sqrt{\frac{\pi}{6}} \frac{k_{\mathrm{B}} T}{\kappa_{\mathrm{eff}}} \sigma\left(R_{\mathrm{a}}-R_{\mathrm{b}}\right)
$$

With this theoretical background, the results shown in Figure 1 are no longer surprising and are in line with eq 7. An increase in the surfactant chain length from $\mathrm{C}_{8} \mathrm{E}_{3}$ to $\mathrm{C}_{12} \mathrm{E}_{5}$ changes $\phi_{\mathrm{C}}^{0}$ and $\phi_{\mathrm{C}}$, whereas the parameters $\sigma, R_{\mathrm{a}}$, and $R_{\mathrm{b}}$, which characterize the polymer, remain the same. According to eq 7, the quotient $\phi_{\mathrm{C}} / \phi_{\mathrm{C}}^{0}$ has to stay constant under these conditions. Evaluating the phase diagrams shown in Figure 1 , one indeed finds that $\phi_{\mathrm{C}} / \phi_{\mathrm{C}}^{0} \approx 0.6$ for all three systems. Thus, the efficiency-boosting effect is the same for all three systems if we refer to the relative decrease in the surfactant volume fraction at the $X$-point. Moreover, we can deduce from eq 8 that a symmetric block copolymer with $R_{\mathrm{a}}=R_{\mathrm{b}}$ does not influence the spontaneous curvature of the interfacial layer. This is exactly what is observed in Figures 2-4 in which the temperature $\tilde{T}$ of the $X$-point is nearly unaffected by the presence of the polymer up to a polymer mass fraction of $\delta=0.16$. Let us now address the question of how polymer concentration, size, structure, and mixtures influence the phase behavior of the ternary base system.

4.2. Polymer Concentration. In the $T(\gamma)$ sections through the phase prism that have been shown thus far, the surfactant/polymer mixture has been considered a pseudocomponent, and therefore the four-component mixture of $\mathrm{H}_{2} \mathrm{O}-n$-alkane $-\mathrm{C}_{i} \mathrm{E}_{j}-\mathrm{PEPX}-\mathrm{PEOY}$ has been considered a pseudoternary system. However, for an exact representation of the phase behavior, a phase tetrahedron (at constant temperature), rather than a phase prism, has to be used. Figure 9 shows a section through the phase tetrahedron of the system $\mathrm{H}_{2} \mathrm{O}-n$-decane $-\mathrm{C}_{10} \mathrm{E}_{4}-\mathrm{PEP} 5-$ PEO5 at a constant water-to-water/oil ratio of $\phi=0.50$, and the mean temperature of the $X$-point $\tilde{T}=31.0{ }^{\circ} \mathrm{C}$. Note that the abscissa corresponds to the ternary base system $\mathrm{H}_{2} \mathrm{O}-n$-decane $-\mathrm{C}_{10} \mathrm{E}_{4}$. Thus, starting at the lefthand side of the abscissa, that is, at $\gamma=0$, and increasing the surfactant concentration continuously, one first

\section{PEP5-PEO5}

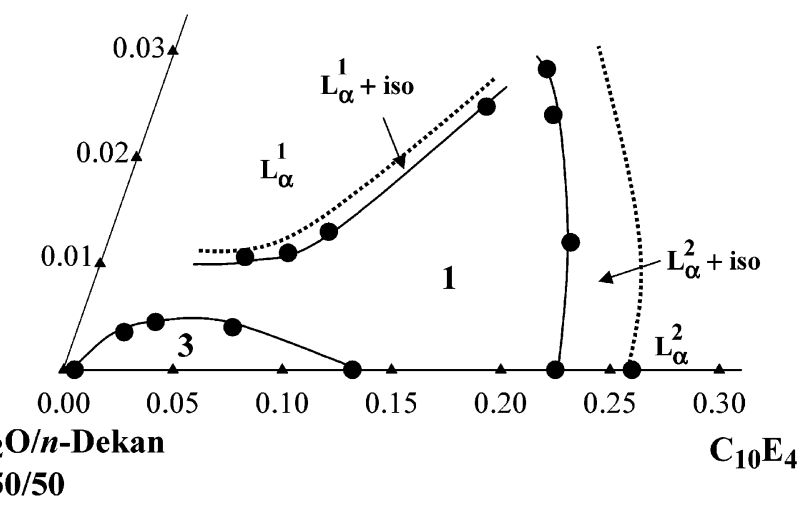

Figure 9. Section through the phase tetrahedron of the system $\mathrm{H}_{2} \mathrm{O}-n$-decane- $\mathrm{C}_{10} \mathrm{E}_{4}-\mathrm{PEP} 5-\mathrm{PEO} 5$ at $\phi=0.50$ and $T=31.0$ ${ }^{\circ} \mathrm{C}$, which corresponds to the mean temperature of the X-point $\tilde{T}$.

reaches a two-phase region (not shown) followed by a threephase region (3) in which a bicontinuous microemulsion coexists with a water and an oil excess phase. At surfactant mass fractions larger than $\tilde{\gamma}$, that is, at $\gamma>0.13$, an extended one-phase region is found until, at $\gamma>0.23$, a concentrated $\mathrm{L}_{\alpha}$ phase $\mathrm{L}_{\alpha}^{2}$ coexists with the bicontinuous microemulsion $\left(\mathrm{L}_{\alpha}^{2}+\right.$ iso). Finally, at $\gamma>0.26$, only the $\mathrm{L}_{\alpha}^{2}$ phase exists.

After adding the amphiphilic block copolymer PEP5PEO5 to the ternary system, one sees that the extension of the three-phase region decreases while that of the onephase region increases. Thus, highly diluted microemulsions with length scales of up to $500 \mathrm{~nm}$ can be formulated by using only $0.5 \mathrm{wt} \%$ of PEP5-PEO5. After increasing the polymer concentration further, one observes a diluted, polymer-containing $\mathrm{L}_{\alpha}$ phase $\mathrm{L}_{\alpha}^{1}$, which initially coexists with the bicontinuous microemulsion $\left(\mathrm{L}_{\alpha}^{1}+\right.$ iso) before turning into a single phase. This $\mathrm{L}_{\alpha}^{1}$ phase is what appears at the low surfactant concentrations in Figure 3, and has been referred to as the " $\mathrm{L}_{\alpha}$ island" thus far. With both high surfactant and high polymer concentrations, the diluted and the concentrated $\mathrm{L}_{\alpha}$ phases merge (omitted in Figure 9), resulting in a two-phase region in which $\mathrm{L}_{\alpha}^{1}$ and $\mathrm{L}_{\alpha}^{2}$ coexist. As can be seen in Figure 3, the merging of the two $\mathrm{L}_{\alpha}$ phases occurs between $\delta=0.10$ and 0.12 at surfactant concentrations of $\gamma \geq 0.10$. Thus, the region denoted as $\mathrm{L}_{\alpha}$ at $\delta \geq 0.118$ is a two-phase region in which a diluted (polymer-containing) $\mathrm{L}_{\alpha}^{1}$ and a concentrated (polymer-free) $\mathrm{L}_{\alpha}^{2}$ phase coexist. The $\mathrm{L}_{\alpha}^{2}$ phase does not contain any polymer because the distance between the surfactant monolayers is too small to host the polymer without a considerable loss of entropy. Therefore, the concentrated $\mathrm{L}_{\alpha}^{2}$ phase always coexists with either a polymer-containing $\mathrm{L}_{\alpha}^{1}$ phase $\left(\mathrm{L}_{\alpha}^{1}+\mathrm{L}_{\alpha}^{2}\right)$ or a polymercontaining microemulsion $\left(\mathrm{L}_{\alpha}^{2}+\right.$ iso). In other words, at high surfactant concentrations, the polymer induces a phase separation into a surfactant-rich (low distance between the monolayers) and a surfactant-poor (large distance between the monolayers) phase in which the latter hosts the polymer in either a second $L_{\alpha}$ phase or a bicontinuous microemulsion. The resulting complex phase behavior was studied extensively with ${ }^{2} \mathrm{H}$ NMR spectroscopy and will be discussed in detail in a follow-up paper. Assuming that the block copolymers at the interface are in the mushroom regime, one can again deduce from eq 7 the efficiency increase with increasing polymer concentration that is shown in Figure 9. According to eq 7, only the number density $\sigma$ of the block copolymer PEP5- 
PEO5 increases with increasing polymer concentration, which, in turn, reduces $\phi_{\mathrm{C}}$.

4.3. Polymer Size and Polymer Symmetry. Polymer Size. As already mentioned in Section 3.3, an increase in the polymer size at a constant polymer fraction $\delta$ does not influence the efficiency-boosting effect. A look at eq 7 provides us with an explanation for this observation. Increasing the polymer size from PEP5-PEO5 to PEP10PEO10 at a constant $\delta$ has two effects. First, it reduces the polymer density $\sigma$ (number density per unit area) at the interface, and second, it increases the factor $\left(R_{\mathrm{a}}{ }^{2}+\right.$ $R_{\mathrm{b}}{ }^{2}$ ). According to eq 7, we induce two opposite effects: the first decreases the efficiency, whereas the latter is expected to increase it. These two opposite effects obviously even out the influence the polymer size has on the efficiency. From a theoretical point of view, eq 7 describes the stability limits of both the bicontinuous microemulsion and the $\mathrm{L}_{\alpha}$ phase. Thus, one would expect an equally insignificant effect of the polymer size on the formation of the $\mathrm{L}_{\alpha}$ phase. Comparing Figures 3 and 4 , however, one sees that this is only the case up to $\delta=0.05$. At higher polymer fractions $\delta$, the stability of the $\mathrm{L}_{\alpha}$ phase is strongly affected by varying the polymer size. This is because, at a given surfactant concentration $\phi_{\mathrm{C}}$, the domain sizes of an $\mathrm{L}_{\alpha}$ phase $\left(\xi_{\mathrm{L} \alpha}\right)$ and a microemulsion $\left(\xi_{\mu \mathrm{E}}\right)$ are not equal. These values are defined as

$$
\xi_{\mathrm{L} \alpha}=\frac{v_{\mathrm{c}}}{a_{\mathrm{c}}} \frac{1}{\phi_{\mathrm{C}}}
$$

and

$$
\xi_{\mu \mathrm{E}}=6 \frac{v_{\mathrm{c}}}{a_{\mathrm{c}}} \frac{\phi(1-\phi)}{\phi_{\mathrm{C}}}
$$

in which $v_{\mathrm{c}}$ and $a_{\mathrm{c}}$ are the surfactant's volume and headgroup area, respectively. Comparing eqs 9 and 10 for equal volumes of water and oil, that is, $\phi=0.5$, one obtains

$$
\xi_{\mu \mathrm{E}}=\frac{3}{2} \xi_{\mathrm{L} \alpha}
$$

Thus, the distance between the surfactant monolayers in the bicontinuous phase is larger than the respective distance in an $\mathrm{L}_{\alpha}$ phase by a factor of three-halves. In other words, the larger the polymer is, the more favorable the formation of a bicontinuous microemulsion becomes. This is exactly what is seen for $\delta=0.12$ in Figures 3 and 4 in which the $\mathrm{L}_{\alpha}$ phase of the PEP5-PEO5 system is spread over a broad concentration range, whereas the corresponding PEP10-PEO10 system only forms a small $\mathrm{L}_{\alpha}$ island surrounded by a bicontinuous microemulsion.

Polymer Symmetry. Comparing Figures 5 and 4, one sees that the polymer symmetry at a given polymer mass fraction and polymer molecular weight influences neither the efficiency nor the extent to which lyotropic liquid crystals are formed. However, the mean temperature $\tilde{T}$ is slightly affected, and the nature of the lyotropic mesophases is strongly affected. Again, we can explain the first observation with eq 7. A change in the symmetry for a given $\delta$ changes neither the number density of the polymer $\sigma$ nor the factor $\left(R_{\mathrm{a}}^{2}+R_{\mathrm{b}}{ }^{2}\right)$. What is changed, however, is the curvature of the interfacial layer, which is expressed in eq 8. The larger the hydrophilic/hydrophobic block size $R_{\mathrm{a}} / R_{\mathrm{b}}$ is, the more positive/negative the curvature becomes, which shifts the mean temperature $\tilde{T}$ toward higher/lower values compared to those of the symmetric reference system. Considering the nature of the lyotropic liquid crystals formed, one could presume that polymers with a large hydrophilic block favor the formation of normal lyotropic liquid crystals such as $\mathrm{I}_{1}$ and $\mathrm{H}_{1}$, whereas polymers with a large hydrophobic block support the formation of the inverse liquid crystalline phases. Looking at Figure 5, however, the exact opposite is observed: whereas with the hydrophilic PEP5-PEO15, the inverse phases $\mathrm{H}_{2}$ and $\mathrm{I}_{2}$ are formed, the $\mathrm{H}_{1}$ and the $\mathrm{I}_{1}$ phases appear with the hydrophobic PEP15-PEP5. This unexpected result can be explained by assuming that the phase behavior of liquid crystals is less temperature dependent than that of microemulsions. For the more hydrophilic polymer, the temperature range in which the bicontinuous microemulsion is formed is shifted toward higher temperatures. We assume that the $\mathrm{I}_{1}$ and $\mathrm{H}_{1}$ phases are part of the phase region denoted as 2 . (Note that we did not study this part of the phase diagram in detail because the phase separation takes at least a couple of weeks. Thus, we cannot exclude that this region consists of more than two coexisting phases, one of which might be an $\mathrm{I}_{1}$ or an $\mathrm{H}_{1}$.) The opposite holds true for the hydrophobic polymer. Another point worth mentioning is the structure of the observed cubic phases. A continuous decrease of the interfacial curvature leads to the following sequence of lyotropic liquid crystalline phases: $\mathrm{I}_{1} \rightarrow \mathrm{H}_{1}$ $\rightarrow \mathrm{V}_{1} \rightarrow \mathrm{L}_{\alpha} \rightarrow \mathrm{V}_{2} \rightarrow \mathrm{H}_{2} \rightarrow \mathrm{I}_{2}$. According to the change in curvature with temperature, $I_{1}$ and $I_{2}$, rather than $V_{1}$ and $\mathrm{V}_{2}$, are expected to appear in the phase diagrams seen in Figures 5-7. Experimental evidence, however, is still missing.

4.4. Polymer Mixtures. Looking at Figure 8, one sees that the 1:1 mixture of PEP5-PEO15 and PEP15-PEO5 behaves like the corresponding symmetric PEP10-PEO10 with regard to the efficiency-boosting effect and the phase boundaries, including the mean temperature $\tilde{T}$. Referring to eqs 7 and 8, one can argue that the average end-to-end distances of the mixed hydrophilic and the mixed hydrophobic blocks, respectively, equal those of the corresponding pure blocks. Thus, the efficiency and the effective spontaneous curvature are not affected by the mixture. In this regard, the absence of the $\mathrm{L}_{\alpha}$ island at low surfactant concentrations is unexpected. Although this phenomenon has not yet been described quantitatively, a qualitative explanation can be given in a schematic drawing, which is seen in Figure 10.

It is reasonable to argue that a mixture of oppositely shaped polymers (top panel) induces a mean curvature of zero and a negative Gaussian curvature, which leads to the formation of a bicontinuous structure. In contrast, a symmetric polymer (bottom panel) is expected to induce both a spontaneous and a Gaussian curvature of zero, thus favoring an $\mathrm{L}_{\alpha}$ structure. Thus, the local, not the mean, curvature needs to be controlled to control the formation of $\mathrm{L}_{\alpha}$ phases in polymer-containing microemulsions.

\section{Conclusions}

We studied the influence of amphiphilic block copolymers on the phase behavior of ternary water-oilsurfactant systems. The focus of this study was to determine how one could optimize the efficiency-boosting effect while simultaneously controlling the formation of lyotropic liquid crystals. For that purpose we chose a model system, namely, water- $n$-decane $-\mathrm{C}_{10} \mathrm{E}_{4}$, and systematically varied the polymer concentration, size, and symmetry. The influence of one particular polymer mixture was studied as well. The following main conclusions can be drawn: 

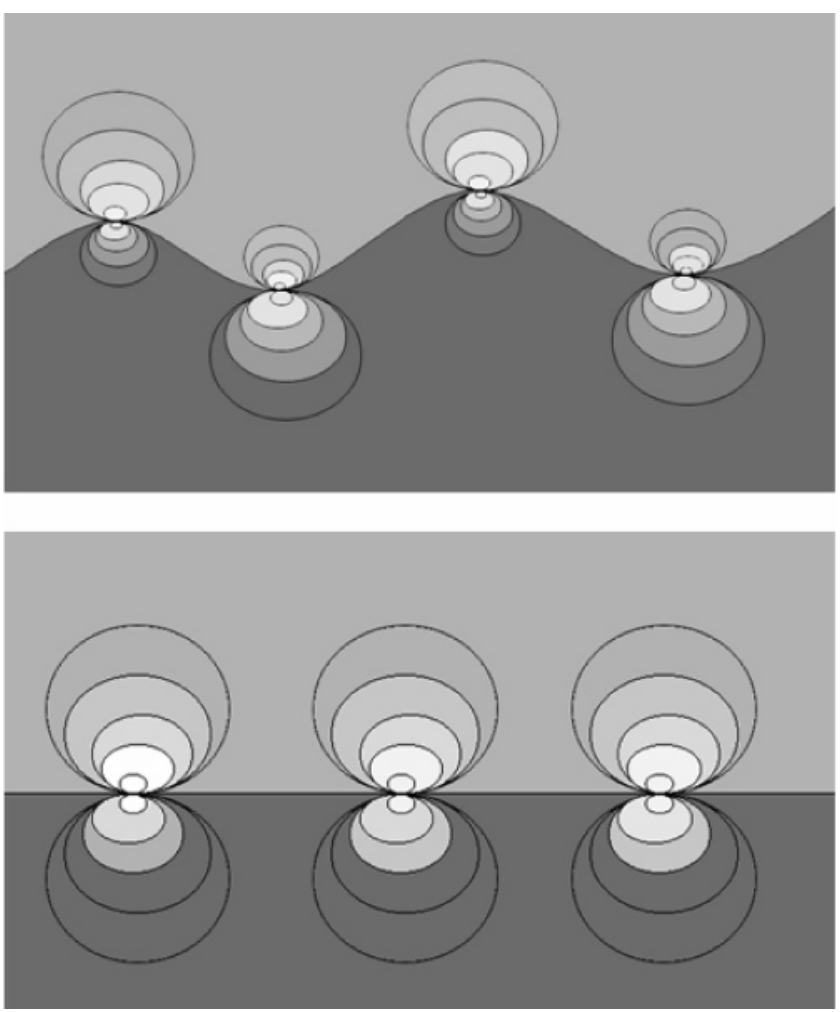

Figure 10. Schematic drawing of amphiphilic block copolymers anchored to a surfactant membrane. Top: oppositely shaped polymers; bottom: symmetric polymers.

Polymer Concentration. For a given polymer, the efficiency-boosting effect increases with increasing polymer concentration. This observation can be explained by eq 7, which says that an increasing number density $(\sigma)$ of the block copolymer at the water/oil interface reduces the surfactant concentration needed to solubilize equal amounts of water and oil $\left(\phi_{\mathrm{C}}\right)$. The increase in efficiency is accompanied by an increase in the tendency to form $\mathrm{L}_{\alpha}$ phases. An increase in the polymer concentration leads to the appearance of an $\mathrm{L}_{\alpha}$ island $\left(\mathrm{L}_{\alpha}^{1}\right)$ at low surfactant concentrations, which finally merges with the concentrated $\mathrm{L}_{\alpha}$ phase $\left(\mathrm{L}_{\alpha}^{2}\right)$. Thus the presence of the polymer induces a phase separation into a surfactant-poor and a surfactant-rich $L_{\alpha}$ phase, the former serving as the host for the polymer.
Polymer Size. An increase in the polymer size at a constant polymer fraction $(\delta)$ does not influence the efficiency-boosting effect, although it has a significant effect on the formation of $\mathrm{L}_{\alpha}$ phases. The first observation is interpreted as the interplay of two opposite effects, namely, a reduction in the polymer density $\sigma$ (number density per unit area) at the interface and an increase in the factor $\left(R_{\mathrm{a}}{ }^{2}+R_{\mathrm{b}}{ }^{2}\right)$, which almost even out the influence the polymer size has on the efficiency. The strong influence the polymer size has on the stability of the $\mathrm{L}_{\alpha}$ phase was attributed to the smaller domain size of the $L_{\alpha}$ phase compared to the bicontinuous microemulsion: the larger the polymer is, the more favorable the formation of a bicontinuous microemulsion becomes.

Polymer Symmetry. The variation of the block sizes influences, first and foremost, the nature of the lyotropic liquid crystals that are observed. The more hydrophobic the polymer is, the more favored normal liquid crystals, such as $\mathrm{H}_{1}$ and $\mathrm{I}_{1}$, become. At first sight, this result seems to be unreasonable because hydrophobic polymers are expected to induce negative interfacial curvatures, thus supporting the formation of the inverse liquid crystals. However, an explanation is offered, namely, that the phase behavior of liquid crystals is less temperature dependent than that of microemulsions. If this holds true, the "expected" liquid crystals are part of the two-phase regions 2 and $\overline{2}$, which were not studied in detail. Another effect the polymer symmetry has on the phase behavior is a slight shift of the mean temperature $\tilde{T}$ : the larger the hydrophilic/hydrophobic block size is, the higher/lower $\tilde{T}$ becomes compared to the symmetric reference system.

Polymer Mixtures. Only with a polymer mixture can the $\mathrm{L}_{\alpha}$ island appearing at low surfactant and high polymer concentrations in the pure polymer systems be suppressed while not affecting the efficiency-boosting effect. Thus, mixing asymmetric polymers of large molecular mass is the most promising way to tune the phase behavior of microemulsion systems. Not only can one achieve an enormous increase in the efficiency, but one can also control $\mathrm{L}_{\alpha}$ formation by mixing appropriate polymers.

Acknowledgment. C.S. is indebted to the DFG and the Fond der Chemischen Industrie for financial support. Illuminating discussions with C. Schmidt are gratefully acknowledged.

\section{LA051463R}

